\title{
Historique de la Congrégation des Missionnaires de \\ l'Immaculée-Conception et des origines de la Société des Missions-Étrangères de Québec
}

\section{Madeleine Loranger}

Volume 38, 1971

La genèse de la Société des Missions-Étrangères de la province de Québec

URI : https://id.erudit.org/iderudit/1007266ar

DOI : https://doi.org/10.7202/1007266ar

Aller au sommaire du numéro

Éditeur(s)

Les Éditions Historia Ecclesiæ Catholicæ Canadensis Inc.

ISSN

0318-6172 (imprimé)

1927-7067 (numérique)

Découvrir la revue

Citer cet article

Loranger, M. (1971). Historique de la Congrégation des Missionnaires de

l'Immaculée-Conception et des origines de la Société des Missions-Étrangères

de Québec. Sessions d'étude - Société canadienne d'histoire de l'Église catholique,

38, 71-84. https://doi.org/10.7202/1007266ar

Tous droits réservés @ Les Éditions Historia Ecclesiæ Catholicæ Canadensis Inc., 1972
Ce document est protégé par la loi sur le droit d'auteur. L'utilisation des services d’Érudit (y compris la reproduction) est assujettie à sa politique d'utilisation que vous pouvez consulter en ligne. 


\section{Historique de la Congrégation des Missionnaires de l'Immaculée-Conception et des origines de la Société des Missions-Étrangères de Québec}

\section{INTRODUCTION}

Après avoir offert nos sincères félicitations et nos vœux fraternels à la Société jubilaire, nous tenons à remercier cordialement les membres de la Société canadienne d'Histoire de l'Église catholique de nous avoir invitées à participer à ce Congrès.

C'est à titre de première congrégation missionnaire fondée au Canada et aussi à cause des liens étroits qui nous unissent à la Société des Missions Etrangères que l'invitation nous a été adressée. Dans un bref exposé, nous vous ferons connaître celle qui a été à l'origine des deux premières fondations missionnaires canadiennes. En terminant, nous survolerons à vol d'oiseau les réalisations apostoliques de la Congrégation des Sceurs Missionnaires de l'Immaculée-Conception depuis sa fondation en juin 1902.

\section{I - A L'ORIGINE DE LA CONGREGATION : LA FONDATRICE}

C'est une femme de chez nous, Délia Tétreault, qui est à l'origine du premier institut missionnaire canadien. Elle naquit le 4 février 1865 à Sainte-Marie-de-Monnoir, aujourd'hui Marieville, au diocèse de SaintHyacinthe.

Une flamme apostolique s'alluma très tôt dans l'âme de la fillette. Toute jeune, elle lisait avec avidité les annales françaises de la Propagation de la Foi et de la Sainte-Enfance. Les récits missionnaires qui peuplèrent alors sa jeune imagination la marquèrent à jamais. Cependant, comme elle le dit elle-même, sa pensée enfantine n'allait pas encore audelà d'une grande admiration pour les religieux et religieuses engagés dans l'apostolat. Mais l'amour suscité dans son cœur pour l'Église missionnaire la tiendra plus tard sans cesse à l'écoute des besoins et des appels des missions.

A l'âge de 18 ou 19 ans, $M^{110}$ Tétreault entra chez les Sœurs de la Charité de Saint-Hyacinthe. Une épidémie de fièvre s'étant déclarée au couvent, ses parents la ramenèrent au foyer. C'est pendant ce séjour de Délia au postulat des Søurs de la Charité que le Seigneur commença à manifester sur elle ses volontés, relativement à deux œuvres missionnaires. 


\section{Délia cherche sa voie}

Vers l'âge de 24 ans, $M^{11 \bullet}$ Tétreault fit la rencontre d'un Jésuite français, le père Almire Pichon. Celui-ci avait fondé à Montréal une maison d'œuvres portant le nom de Béthanie dont il avait confié la direction à l'une de ses compatriotes, madame Poitou. Le dessein du père Pichon était d'établir une véritable communauté. Ayant connu $\mathrm{M}^{110}$ Tétreault, il lui parla du projet qu'il nourrissait, et lui demanda sa coopération. La jeune fille, croyant que ce pourrait être là l'œuvre à laquelle Dieu l'appelait, accepta courageusement, bravant les oppositions qui contrecarraient sa décision.

La maison affectée à l'œuvre du père Pichon était située dans un quartier pauvre de Montréal. Durant dix ans, $M^{110}$ Tétreault s'y dévouera sans compter par toutes sortes d'œuvres auprès des pauvres, spécialement par l'apostolat catéchétique et marial. C'est en remplissant ce rôle qu'elle fit la connaissance de $\mathrm{M}^{110}$ Joséphine Montmarquet, de Montréal, qui devait devenir plus tard sa première collaboratrice. Dès l'abord, ces deux âmes se comprirent et se lièrent d'amitié.

Cependant, $\mathbf{M}^{110}$ Tétreault - elle-même le révéla dans la suite était sans cesse hantée par la pensée des missions et poussée à fonder une communauté pour leur venir en aide. Sentant son incapacité et craignant l'illusion, elle croyait sage de chasser cette pensée tenace. Un jour qu'elle était assise dans le jardin de la maison paternelle, elle éprouva l'action de l'Esprit-Saint en son âme et s'y livra dans un acte de confiant abandon ${ }^{1}$.

Durant un voyage que le père Pichon fit en France, $M^{110}$ Tétreault prit la liberté de dévoiler à $M$. l'abbé Gustave Bourassa ses perplexités. Constatant sans doute que sa dirigée était guidée par l'esprit de Dieu, $M$. l'abbé Bourassa l'engagea à faire part de ses angoisses à l'archevêque de Montréal. $M^{\mathrm{gr}}$ Bruchési écouta, réfléchit, pria, consulta le savant et saint M. Charles Lecoq, sulpicien. Celui-ci, après une entrevue avec la jeune fille, répondit à monseigneur qu'il pouvait procéder sans crainte. Quelque temps après, $\mathrm{M}^{\mathrm{gr}}$ Bruchési écrivait à l'abbé Bourassa: " Continuez à voir $M^{110}$ Tétreault, je crois que Dieu veut que vous vous occupiez de l'œuvre à laquelle elle désire consacrer sa vie ${ }^{2}$. .

$M^{\text {lle }}$ Tétreault jette les bases de la Congrégation des M.I.C.

L'établissement d'une école apostolique - première dénomination de notre Communauté - fut approuvé le 12 janvier 1901. Au début de 1902 , nous voyons la future fondatrice, à peine guérie d'une grave mala-

1 Archives M.I.C., Fil. $\mathrm{n}^{\circ} 1$, Doc. $\mathrm{n}^{\circ}$ 8, M. le chanoine Roch.

2 Ibid., Fil. M ${ }^{\text {gr }}$ Bruchési, 2/6/1901. 
die, prendre de nouvelles initiatives pour faire éclore cette œuvre dont le but était d'aider les missions. Le 24, février 1902, elle loue une maison au numéro 900, avenue Maplewood, quartier de la Côte-des-Neiges et, le 3 juin suivant, elle en prend possession avec ses deux premières collaboratrices : Joséphine Montmarquet et Ida Lafricain.

\section{L'épreuve}

La jeune institution aura bientôt l'occasion de prouver son souci missionnaire et son total abandon par un double sacrifice. Deux ans après la fondation, soit le 17 mars 1904, $\mathbf{M}^{\mathrm{gr}}$ Adélard Langevin, archevêque de Saint-Boniface, demanda et obtint un membre du trio initial, $\mathbf{M}^{\mathbf{1 1}}$ Ida Lafricain, pour aller prendre la direction de la communauté en formation des Missionnaires Oblates du Sacré-Cœur et de Marie-Immaculée, au Manitoba. Puis M. l'abbé Gustave Bourassa, le dévoué directeur de l'École Apostolique, fut victime d'un accident dont il mourut quelques semaines plus tard, le 20 novembre 1904, laissant dans une grande désolation la fondatrice et sa jeune famille spirituelle. Elles crurent que c'en était fait de l'œuvre projetée ... .

\section{$M^{\mathrm{gr}}$ Bruchési remplace le père disparu...}

$\mathrm{Au}$ lendemain de cette chute qui devait être fatale, $\mathbf{M}^{\mathrm{gr}}$ Bruchési partait pour Rome où il devait participer au cinquantenaire de la proclamation du dogme de l'Immaculée-Conception. Cloué sur un lit de douleur, M. l'abbé Bourassa avait prié le prélat de parler de l'œuvre naissante au Souverain Pontife. Que s'était-il passé entre Dieu et son serviteur? " Je l'ignore», disait plus tard $\mathrm{M}^{\mathrm{gr}}$ Bruchési dans l'homélie de la première profession; " mais ce que je sais, c'est que je compris que je devais remplacer le père qui vous était enlevé. C'est le 30 novembre que je parlai de vous pour la première fois au Saint-Père. Après avoir simplement exposé les faits, je dis : "Saint Père, je ne demande pas, je ne discute pas; décidez et votre serviteur obéira. 》 Pie $\mathrm{X}$ me jetant un regard doux et pénétrant répondit : « Fondez, Monseigneur, et la bénédiction de Dieu descendra sur cette nouvelle fondation ${ }^{3}$."

Quelques jours plus tard, le 7 décembre 1904, dans une seconde audience à l'archevêque de Montréal, Pie X donnait lui-même à la congrégation le nom de Société des Sœurs Missionnaires de l'Immaculée-Conception.

Un câblogramme portant l'heureuse nouvelle fut adressé de Rome à $\mathrm{M}^{\mathrm{gr}}$ Racicot, vicaire général, et transmis à la petite association.

L’École Apostolique devenait donc un institut religieux voué aux missions étrangères.

$3 \quad$ Ibid., Fil. $\mathrm{n}^{\circ} 1$, Doc. $\mathrm{n}^{\circ} 47,8 / 8 / 1905$. 
A quelques jours de là, $\mathrm{M}^{\mathrm{gr}}$ Bruchési écrivait de Rome à Mère Sainte-Anne-Marie, C.N.D., supérieure au Mont-Sainte-Marie, et grande amie de l'œuvre à ses débuts: "Que $\mathrm{M}^{110}$ Tétreault s'abandonne à la Providence et qu'elle remercie Dieu; elle aura au ciel son protecteur d'autrefois, et dans l'Archevêque de Montréal, ici-bas, un père et un ami dévoué. Le 30 novembre dernier, j'ai parlé d'elle au Saint-Père ainsi que de son auvre actuelle et de ses projets d'avenir. J'ai fondé ce jour-lä, avec l'entière approbation et les bénédictions de $\mathrm{Pie} X$, l'Institut qu'elle avait rêvé pour les missions étrangères et j'ai même reçu de Sa Sainteté le nom que cet Institut doit porter ${ }^{4}$. \$

Cette date du 30 novembre est encore pour nous aujourd'hui la fête par excellence de la RECONNAISSANCE. Toutes les familles religieuses ont une spiritualité propre, inspirée directement du cour d'un saint, fondateur ou fondatrice. La nôtre, Délia Tétreault, était obsédée pour ainsi dire, par la pensée de l'extension du règne du Christ aux confins du monde, dans un esprit d'action de grâces. * Travailler aux intérêts sacrés de Dieu par tous les moyens à notre portée et le remercier sans cesse pour nous-mêmes et pour tous les hommes, voilà, en deux mots, la fin exacte de notre œuvre. " Ces lignes jettent une plus vive lumière sur l'idée qui formera le cœur de sa spiritualité: en action de grâces missionnaires!

Il faut signaler aussi le caractère essentiellement marial de la fondation de mademoiselle Tétreault. Cette vocation apostolique inspirée par la reconnaissance ne se réalise pleinement, dans sa pensée, que sous l'égide de Marie. Elle s'adonna durant plusieurs années à la propagation de la dévotion mariale selon saint Louis-Marie de Montfort et elle voulut ardemment que toutes ses filles spirituelles soient pénétrées d'un amour indéfectible pour l'Immaculée, patronne de la Congrégation. Tous ses écrits en témoignent, le nom de Marie revient constamment sous sa plume. Nous retrouvons dans la citation suivante les trois dimensions caractéristiques de la spiritualité qu'elle a insufflée à sa communauté : "L'apostolat auprès des infidèles nous a été donné, me semble-t-il, par la Sainte Vierge, comme moyen extérieur de manifester notre reconnaissance ${ }^{5}$.

\section{PREMIÈRE PROFESSION RELIGIEUSE}

Le 8 août 1905, les deux premiers membres de l'Institut firent profession. Délia Tétreault prit le nom de Sœur Marie du Saint-Esprit et Joséphine Montmarquet celui de Sœur Saint-Gustave en souvenir de M. l'abbé Gustave Bourassa. Ce même jour, trois aspirantes commencèrent leur noviciat.

4 Ibid., Fil. $\mathrm{n}^{\circ} 1$, Doc. $\mathrm{n}^{\circ} 30,31 / 12 / 1904$.

5 Le Précurseur, janvier-février 1965, p. 312-314. 


\section{Premier départ pour les missions}

Tout en préparant à l'apostolat les aspirantes qui se joignaient à elle, la jeune famille religieuse attendait dans la prière et le travail le moment fixé par Dieu pour l'essor de ses premières missionnaires.

Au printemps de $1908, \mathrm{M}^{\mathrm{gr}}$ Jean-Marie Mérel, préfet apostolique de Canton, Chine, visitant à Montréal quelques confrères chez les Sulpiciens, apprit par eux l'existence d'un institut missionnaire canadien nouvellement fondé et il voulut faire sa connaissance. L'exposé des besoins de l'immense préfecture du Kouang Tong remplit d'enthousiasme les futures apôtres. Le visiteur rencontra $\mathbf{M}^{\mathrm{gr}}$ Bruchési qui se dit heureux d'offrir à la Chine les prémices de ses missionnaires.

Peu de temps après cette visite, $M^{g r}$ l'archevêque étant allé à Rome, demanda au cardinal Gotti, de la Congrégation de la Propagande, vers quelles contrées il serait préférable d'orienter ses missionnaires, - Tous les pays de missions vous seront ouverts, lui fut-il répondu. Aucune contrée ne vous est assignée en particulier. Il vous est dit comme autrefois aux apôtres : "Allez, intruisez toutes les nations ${ }^{6}$." Aussitôt revenu au Canada, monseigneur, de concert avec la fondatrice, choisit les six privilégiées qui partiraient pour Canton. $\mathbf{M}^{\text {sr }}$ Mérel pressait le départ : Q Que vos religieuses viennent partager notre pauvreté et nos travaux,... , écrivait le préfet apostolique à Mère Marie du SaintEsprit. "Leurs ouvres seront un épanouissement de votre florissante Eglise de Montréal, un nouveau fleuron de sa couronne ${ }^{7}$. D

Le jour du départ, 8 septembre 1909, $M^{\text {gr }}$ Bruchési avait voulu, pour développer l'esprit missionnaire chez ses diocésains, que la cérémonie d'adieu ait lieu à la cathédrale et qu'on lui donnât tout l'éclat possible: c'était la première fois que le Canada voyait partir des missionnaires d'un institut national.

A la fin de cet exposé, nous jetterons un coup d'œil sur les réalisations de notre congrégation durant ses soixante-neuf années d'existence.

Auparavant, nous croyons répondre au désir de nos auditeurs en faisant connaître l'origine de la parenté apostolique qui existe entre la Société des Missions Étrangères et la nôtre. C'est en nous appuyant sur des données historiques tirées de nos archives que nous parlerons de la genèse de la Société jubilaire.

\section{II - LA SOCIÉTÉ DES MISSIONS ÉTRANGÈRES DE QUÉBEC}

Au début du présent exposé nous avons parlé du séjour que fit $M^{110}$ Tétreault au postulat des Sœurs de la Charité de Saint-Hyacinthe et

6 Archives M.I.C., Fil. M ${ }^{\mathrm{gr}}$ Bruchési, 7/11/1908.

7 Ibid., Fil. évêques de Canton, 1/2/1909. 
nous avons fait allusion à une communication de caractère surnaturel qu'elle aurait eue à cette époque, 1883-1884.

«Un soir, a-t-elle spécifié, que j'étais à travailler avec des postulantes dans une petite pièce, il me sembla entendre que je devais plus tard fonder une congrégation de femmes pour les missions et travailler à la fondation d'une société d'hommes semblable, un séminaire des missions étrangères sur le modèle de celui de Paris. »

Cette confidence avait été faite par Mère Marie du Saint-Esprit à Mère Saint-Gustave, sa première compagne et collaboratrice dans la fondation de notre institut. Celle-ci l'avait notée de sa main dans un simple cahier brouillon conservé aux archives. Dans ce cahier, nous trouvons aussi les lignes suivantes sur le même sujet :

- La même année ou l'année suivante de celle où j'eus l'idée d'une congrégation de femmes missionnaires et d'un séminaire des missions étrangères, étant à la campagne chez un oncle, j'allais un jour avec mes cousines cueillir des fruits. Au moment où j'étais occupée de ma cueillette je vis en esprit une grande maison, comme un monastère, qui était peuplée de prêtres et une autre, peuplée de religieuses ${ }^{8}$.

Il est à remarquer que celle qui prenait note de ces confidences, Mère Saint-Gustave, ne vit point l'établissement du séminaire de Pont-Viau puisqu'elle mourut en 1917, quatre ans avant la fondation de la Société des Missions Étrangères.

On lit, dans l'histoire religieuse de notre pays, l'origine des vocations qui poussèrent au Canada une Marie de l'Incarnation, une Jeanne Mance, une Marguerite Bourgeoys, un Monsieur de la Dauversière. Ces fondateurs avaient reçu des impulsions divines qui se réalisèrent dans la suite. N'est-ce pas un peu l'histoire qui se répète ici par des inspirations révélatrices des mystérieux desseins de Dieu sur une humble religieuse de chez nous?

\section{L'autre œuvre}

En même temps que Délia Tétreault jetait les bases de son institut missionnaire féminin, elle songeait à faire surgir l'autre cuvre, comme elle l'appelle, celle des prêtres pour les missions lointaines. Ainsi, nous lisons dans ses écrits personnels qu'en octobre 1901, donc avant même la fondation de notre communauté, elle offrait à la Sainte Vierge son rosaire quotidien, ses souffrances, ses sacrifices pour obtenir l'établissement des deux œuvres ${ }^{\theta}$.

$8 \quad$ Ibid., Fil. $\mathrm{n}^{\circ}$ 1, Doc. $\mathrm{n}^{\circ}$ 7, p. 5-8.

9 Ibid., Fil. $\mathrm{n}^{\circ}$ 1, Doc. $\mathrm{n}^{\circ} 112$. 
En 1902, des démarches en vue de la fondation d'un séminaire des missions étrangères étaient commencées auprès de $M^{g r}$ Bruchési puisque celui-ci, durant un voyage en Europe, écrivait à $\mathbf{M}^{110}$ Tétreault:

" J'ai vu à Paris le Supérieur général des Missions Étrangères. La fondation d'un séminaire canadien à Montréal lui paraît rencontrer de grandes difficultés; j'abandonne tout entre les mains de Dieu. ’

En 1903, c'est le père Alphonse-Marie Daignault, jésuite, alors procureur en Angleterre de la mission africaine du Zambèze qui, mis au courant des visées apostoliques de $M^{110}$ Tétreault, lui donnait ce conseil: "Je crois que, pour le moment, vous devez concentrer vos efforts sur votre première fondation. Le bon Dieu éclairera plus tard s'il y a quelque chose à faire ailleurs. "

Deux ans ne s'étaient pas écoulès que Ie père Daignault se montrait plus favorable au projet et, sur la demande de $M^{110}$ Tétreault, plaidait en ces termes la cause du séminaire auprès de $\mathbf{M}^{\mathrm{gr}}$ Bruchési :

"... Le jour viendra peut-être où il vous sera donné de voir la fondation d'un séminaire des missions étrangères. Le jour n'est peut-être pas éloigné où notre cher Canada pourra donner des martyrs au ciel et compter comme une de ses plus belles gloires d'avoir sa part dans la conversion des nations non chrétiennes. A vous, Monseigneur, reviendra la sainte gloire et le grand mérite d'avoir frayé la voie et donné l'exemple ${ }^{10}$.

Vers 1914, Mère Marie du Saint-Esprit faisait à son assistante cette autre confidence :

- Depuis deux ans et depuis quelques mois surtout, cette idée de la fondation d'un séminaire pour les missions étrangères me hante... Elle est accompagnée de paix et me porte au sacrifice. Travailler de tout mon pouvoir à la fondation de ce séminaire me semblerait être le complément de ma vocation. Si j'allais entreprendre un voyage en Chine ou toute autre chose où ma vie pourrait être menacée, je craindrais de mourir avant que cette œuvre fût lançée. Elle devrait être entreprise secrètement et il ne faudrait pas qu'on sût qu'une femme s'y intéresse ${ }^{11}$... *

Avec le temps, des lumières surgiront au sujet de l'œuvre du séminaire et Mère Marie du Saint-Esprit jettera les notes suivantes pêle-mêle, au fil de la pensée, sur un bout de papier :

- Il me semblait, durant la messe, que le bon Dieu ne voulait pas que cette nouvelle æuvre canadienne soit greffée sur un vieil arbre et que c'était du neuf qu'il désirait. Saint François Xavier, quand il par-

10 Ibid., Lettres: $\mathrm{M}^{\mathrm{gr}}$ Bruchési (1903); père Daignault (1905).

11 Ibid., Fil. $n^{\circ}$, Doc. $n^{\circ} 7$, p. 6. 
tit pour les Indes, continuait-elle, n'avait aucune expérience personnelle, il n'avait pas en main non plus les méthodes de ses devanciers; il ne voulait que faire connaître Dieu et sauver les âmes, sans aucun mélange d'intérêt, libre de toute considération humaine, de nationalité, et même d'intérêt personnel. Réunissons des jeunes gens ayant la flamme du zèle, formons-en des saints et nous aurons de bons missionnaires. »

Suivant toujours son plan, elle poursuit :

"Au soir du 2 janvier, sans qu'aucune pensée ne l'ait provoquée, l'idée de la fondation d'une société de prêtres pour les missions étrangères, ou plutôt le plan de cette fondation se présenta à mon esprit d'une manière claire et précise: cette société serait nationale. Avec l'autorisation de $\mathbf{M}^{\mathrm{gr}}$ l'archevêque, le plan de l'œuvre projetée serait exposé aux évêques de la province en leur demandant un prêtre de leur diocèse respectif pour former le noyau de la nouvelle association. Pour ce qui est du soutien de l'œuvre, les recettes de la Propagation de la Foi, que les prêtres de la société auraient charge de recueillir, y seraient affectées, du moins pour les débuts ${ }^{12} \ldots$.

On trouve encore, dans les écrits de Mère Marie du Saint-Esprit, l'énoncé d'un plan d'organisation, genre de mémoire dont elle se serait servie dans l'occasion pour démontrer l'opportunité de la fondation d'une société pour les missions. Elle apporte d'abord à l'appui les raisons suivantes : premièrement, la diminution des vocations missionnaires à cause de la persécution religieuse qui sévissait en France; ensuite, l'opportunité pour le Canada de payer sa dette de reconnaissance à l'Église pour la foi reçue grâce au zèle des missionnaires, étant maintenant suffisamment pourvu d'institutions religieuses.

En 1919, la maîtresse des novices se trouva présente à la conversation qui s'engagea sur ce sujet entre $\mathbf{M}^{\mathbf{g r}}$ François-Xavier Brunet, évêque de Mont-Laurier, de passage à notre maison de Nominingue, et Mère Marie du Saint-Esprit. Celle-ci émit l'idée, entre autres, que le temps semblait venu pour le Canada français de prendre enfin son rôle comme nation dans l'œuvre missionnaire, faisant remarquer que jusquelà, notre pays avait donné bon nombre de ses enfants, versé des sommes considérables en faveur des contrées non évangélisées et que, néanmoins, il passait pour ne point faire sa part dans l'œuvre des missions... $\mathbf{M}^{\mathrm{gr}}$ Brunet promit d'user de l'influence qu'il pourrait avoir pour faire aboutir le projet qu'il fit sien. "Il faut que cette œuvre réussisse ", ditil ${ }^{13}$.

C'est vers cette époque que Mère Marie du Saint-Esprit obtint de $\mathbf{M}^{\mathrm{gr}}$ Bruchési l'autorisation d'aller plaider elle-même auprès des évêques

12 Ibid., Fil. $n^{\circ} 1$, Doc. $n^{\circ} 112$.

13 Ibid., Mémoires de Sour Saint-Jean-François-Régis, p. 11. 
de la province de Québec la cause de la fondation d'un séminaire, pour obtenir leur coopération. - Tâche humainement lourde et ingrate, mais qui devait finir par porter ses fruits ${ }^{14}$.,

Cependant, le temps s'écoulait et la fondation retardait toujours. Mère Marie du Saint-Esprit invitait toutes ses soeurs, surtout les novices et les malades, à prier pour l'obtention de cette grâce si ardemment désirée. On était en 1920 et la santé de $\mathrm{M}^{\mathrm{gr}}$ Bruchési déclinait d'une façon alarmante. Après avoir fait tout en son pouvoir, supplié sans résultats apparents, une ressource restait : c'était l'immolation. Déjà elle avait usé de cette monnaie de première valeur, mais il semble qu'en 1920 l'offrande fut plus totale que jamais. Avec une intensité de volonté qui ne mettait point de bornes, elle demanda à passer le reste de ses jours dans les humiliations, les mépris de tous genres et, mue par un courage noũveãũ, elle alla ensuite faire un suprême assaut auprès de $\mathbf{M}^{\mathbf{g}}$ Bruchési qui finit par lui dire : « Si vous voulez un séminaire canadien, trouvez-moi des prêtres ....

Peu après, un ecclésiastique revenant à jeun d'un office religieux dans le voisinage, sonnait à la porte du couvent d'Outremont : c'était l'abbé Louis-Adelmar Lapierre. La statue de la Sainte Vierge devant notre maison lui fit penser qu'il s'agissait d'un couvent et que volontiers on lui servirait une tasse de café. Mère Marie du Saint-Esprit se rendit lui souhaiter la bienvenue. Pendant le repas qu'elle lui offrit, l'entretien tomba sur le sujet des missions et le prêtre en vint à déclarer qu'il avait ardemment désiré se consacrer à l'apostolat missionnaire lors de son ordination. Son interlocutrice pressentit que c'était l'envoyé de Dieu et elle lui fit connaître le projet qui s'élaborait, lui conseillant de se présenter à $\mathrm{M}^{\mathrm{gr}}$ Bruchési, ce qu'il fit sans tarder. L'archevêque reconnut que le Seigneur manifestait clairement sa volonté.

\section{L'attente touchait à sa fin.}

Le 2 octobre 1920, $\mathrm{M}^{\mathrm{gr}}$ Jean-Baptiste de Guébriant, de la Société des Missions Etrangères de Paris et vicaire apostolique de Canton, Chine, arrivait à Montréal. Son voyage avait un double but. Le premier était avoué : visiter les Sœurs Missionnaires de l'Immaculée-Conception et traiter avec elles des œuvres auxquelles la communauté se dévouait dans son vicariat; le second était moins connu: s'entendre avec l'épiscopat de la province de Québec pour établir à Montréal une succursale du Séminaire des Missions Étrangères de Paris. De passage à Rome, quelques mois auparavant, il avait obtenu cette autorisation de la Congrégation de la Propagande, au nom de sa Société.

14 Abbé Clovis Rondeau, p.m.é., Mère Marie du Saint-Esprit, L'CEuvre des Tracts, Montréal, décembre 1943, p. 13. 
A son arrivée à Montréal, dans la matinée du 2 octobre, il alla saluer $\mathbf{M}^{\mathrm{gr}}$ Bruchési et prit résidence à la maison mère des Sœurs Missionnaires de l'Immaculée-Conception. Ce même jour, $M^{\mathrm{gr}}$ l'archevêque appelait à Montréal quelques évêques des diocèses avoisinants: nosseigneurs Paul Larocque de Sherbrooke, J. S. Hermann Brunault de Nicolet et Guillaume Forbes de Joliette s'y rendirent. L'entrevue eut lieu dans la soirée à la maison mère d'Outremont et Mère Marie du Saint-Esprit fut invitée à prendre part aux délibérations. Mais toujours sobre de communications en ce qui pouvait la mettre en évidence, elle ne révéla rien de ce qui s'était passé durant la réunion, du moins en ce qui concernait la fondation du séminaire. Pour la raison énoncée plus haut, elle tenait à garder l'incognito au sujet de l'œuvre projetée, autant que faire se pouvait. Tout au plus relevons-nous dans les archives ce souvenir d'une phrase adressée aux sœurs à son arrivée au réfectoire le jour de cette visite mémorable: "Jamais si petite maison n'a vu tant de Grandeurs ! "

Le vicaire apostolique de Canton fit aussi une visite à l'archevêché de Québec. Quitta-t-il le pays avec l'espoir qu'une succursale du séminaire de la rue du Bac y serait bientôt établie ? Il semble que oui.

Peu après, $M^{\mathrm{gr}}$ Bruchési fit des démarches auprès de la Propagande pour connaître la portée de l'autorisation accordée à $\mathrm{M}^{\mathrm{gr}}$ de Guébriant et les possibilités d'une fondation canadienne. Le Préfet, Son Eminence le cardinal Van Rossum, accorda la priorité aux évêques intéressés, qui résolurent alors, en principe, de fonder un séminaire canadien.

Le dernier acte d'administration de $\mathrm{M}^{\mathrm{gr}}$ Bruchési fut la lettre qu'il adressa, le 30 novembre 1920 , à $M^{g r}$ Paul-Eugène Roy, évêque coadjuteur de Québec. En voici un extrait :

«... Je voudrais vous écrire longuement, mon pauvre état de santé ne me le permet pas et je ne vous envoie que quelques lignes après l'entretien que je viens d'avoir avec la Supérieure générale des Sours Missionnaires de l'Immaculée-Conception, pour vous dire que dans cette œuvre de fondation d'un séminaire canadien des missions étrangères, je suis absolument avec Son Eminence et avec vous d'esprit et de cœur ${ }^{15} \ldots$...

L'heure tant souhaitée était enfin venue ... Le 2 février 1921, l'épiscopat de la province civile de Québec décrétait, à l'unanimité, la fondation d'un séminaire canadien des missions étrangères.

$M^{\mathrm{gr}}$ Guillaume Forbes, évêque de Joliette, sacrifia en faveur de l'institution naissante un des plus éminents prêtres de son diocèse, $M$. le chanoine Avila Roch qui devint le supérieur de la Société.

15 Archives M.l.C., Fil. $\mathrm{n}^{\circ}$ 1, Doc. $\mathrm{n}^{\circ}$ 124, $\mathrm{M}^{\mathrm{gr}}$ Bruchési. 
Un terrain fut acheté au bord de la Rivière-des-Prairies, et le Conseil d'administration du nouveau séminaire, avec l'approbation de l'épiscopat, dans un geste de libéralité dont notre congrégation gardera toujours un souvenir reconnaissant, céda à cette dernière, à titre gratuit, une partie de la propriété pour l'érection d'une maison de la communauté.

Le projet que Mère Marie du Saint-Esprit avait toujours considéré comme le complément de sa vocation était réalisé. Elle en rendit grâce au Seigneur et rentra dans le silence et dans l'ombre, assurée que la Société des Missions Étrangères se développerait rapidement sous la tutelle vigilante de ses fondateurs. Elle restera toujours présente à l'œuvre par la prière et la souffrance.

A côté de la "grande maison " qui se peuplera de prêtres, s'élèvera cette autre "peuplée de religieuses », entrevues jadis par Délia Tétreault au temps de la genèse des deux œuvres.

En 1925, le prêtre gagné à la cause des missions grâce à une tasse de café, l'abbé Louis-Adelmar Lapierre, partait, à la tête d'un premier groupe de prêtres pour la lointaine Mandchourie. Il deviendra bientôt chef ecclésiastique de Szepingkai et ne tardera pas à inviter les Missionnaires de l'Immaculée-Conception à partager le labeur apostolique de ses prêtres; huit missions furent successivement acceptées par la communauté entre les années 1927 et 1933.

Depuis lors, les deux familles missionnaires œuvrèrent ensemble, d'abord en Chine, puis aux Philippines, à Cuba et, depuis 1960, au Pérou. Mais c'est surtout en Mandchourie que la fraternité apostolique se scella dans la souffrance partagée au camp de concentration durant la guerre, dans la persécution qui suivit, dans les privations multiples et une pauvreté totale à certaines époques. A côté du chef au courage indomptable, le vénéré $\mathrm{M}^{\mathrm{gr}}$ Lapierre qui décéda en 1952 au milieu de son troupeau qu'il n'avait pas voulu abandonner, les derniers prêtres des Missions Étrangères, et les Missionnaires de l'Immaculée-Conception demeurés en Mandchourie, lutteront jusqu'à l'extrême limite, c'est-à-dire jusqu'à leur expulsion par le parti communiste à la fin de décembre 1953.

Il ne nous appartient pas d'exposer les fastes de l'histoire de la Société des Missions Étrangères; cependant, comme on l'a vu, sa naissance et certaines périodes de son existence ont été liées si intimement à notre propre histoire que nous ne pouvions raconter l'une sans impliquer l'autre également.

\section{III - RÉALISATIONS DE LA COMMUNAUTÉ DES M.I.C.}

Le moment est venu d'exposer brièvement les réalisations apostoliques de notre congrégation. Quand Mère Marie du Saint-Esprit fonda son institut en 1902, et même quand elle nous quitta pour le ciel, le $1^{\text {rr }}$ octobre 1941, il n'existait encore aucune faculté d'études missiologiques 
au Canada. Ce n'est qu'en 1948 que fut créé, à l'Université d'Ottawa, le premier Institut de Sciences missionnaires. Pionnière dans ce domaine, la fondatrice vécut donc par ses propres moyens humains, décuplés par la grâce, la période des difficiles commencements. En étudiant les directives qu'elle a laissées, on découvre chez elle un sens missionnaire qu'on dirait inné, tant son esprit intuitif décèle les meilleurs plans d'action et les moyens les plus efficaces pour les réaliser.

Elle était convaincue, par exemple, qu'il est de première importance pour l'avenir des missions, d'imprégner le peuple d'esprit missionnaire; que, pour aller porter la Bonne Nouvelle aux confins du monde, il faut des apôtres, des recrues pour la relève, des ressources financières. Elle songea donc à faire surgir au pays des centres d'animation missionnaire, des cuvres qui provoqueraient un éveil et un intérêt nouveaux pour les missions. Dans plusieurs diocèses, elle sera autorisée à ouvrir des maisons où ses sœurs travailleront à promouvoir les œuvres pontificales de la Propagation de la Foi et de la Sainte-Enfance. Réalisant l'importance d'un instrument de propagande qui pénétrerait dans des milliers de familles, elle n'hésita pas à se lancer, en 1920, dans la publication d'une revue qui, adaptée aux temps nouveaux, paraît encore : Le Précurseur.

Toujours sous son impulsion, on créa des écoles apostoliques, des ouvroirs destinés à faire participer les laïques au soutien des missions. On ouvrit des hôpitaux, des refuges, des centres d'accueil où se donnaient et se donnent encore des cours de langue pour les Orientaux immigrés au Canada.

Pour intensifier la vie spirituelle et l'amour des missions chez les femmes et les jeunes filles, elle établit plusieurs maisons de retraites fermées, dont celles de Québec, Joliette, Saint-Jean et Nominingue sont encore en activité. La première retraite fermée collective féminine au pays eut lieu le 26 juin 1911, à l'établissement d'Outremont.

Comme nous l'avons déjà dit, c'est en 1909 que s'effectua le départ initial pour les missions. Les premières recrues s'orientèrent vers Canton, Chine, où les attendaient de nombreuses activités : écoles, ateliers, asile de vieillards et d'infirmes, crèches, orphelinats. Une petite association de vierges chinoises catéchistes s'occupait à diffuser la doctrine chrétienne.

En 1913, on accepta l'œuvre de la léproserie de Shek Lung où depuis quelques années un prêtre belge, l'abbé Louis-Lambert Conrardy, son fondateur, se dévouait auprès d'un groupe de lépreux. Ce lazaret abritera à une certaine époque plus de mille malades.

Cette léproserie de Shek Lung, de même que la mission-mère de Canton, l'école européenne de Shameen, la crèche Notre-Dame-de-la-Providence de To Kom Hang, toutes situées en bordure de Canton, subsistèrent jusqu'en 1951 et 1952, alors que le parti communiste chinois s'ap- 
propria ces établissements et en expulsa les missionnaires. C'est à ce moment que cinq missionnaires de l'Immaculée-Conception payèrent par des mois d'emprisonnement en cachots communistes la rançon de leur dévouement au soin de l'enfance abandonnée.

Nos sœurs exilées de la Chine continentale voulurent continuer d'aider ce peuple dont elles connaissaient bien la langue et la culture. C'est dans l'île de Taiwan, que nous appelons Formose ou la Belle, que la plupart d'entre elles se fixèrent et continuent encore leur travail dans six centres d'action religieuse, éducative et sociale.

Un autre contingent d'exilées prit la route des Philippines où la Communauté s'était implantée en 1921 en acceptant la direction d'un hôpital pour la colonie chinoise. Peu à peu, des œuvres d'éducation surgirent aussi et prirent de l'ampleur. Mais la Seconde Guerre mondiale apporta de nouveau ruines et désolation. L'Ácadémie anglo-chinoise forcément abandonnée, les religieuses furent reléguées, comme tant d'autres étrangers, en des camps de concentration. La paix revenue, on recommença à neuf. Actuellement la communauté compte douze établissements dans l'archipel. Le noviciat de Baguio, ouvert en 1955, a vu passer dans ses murs une soixantaine de sœurs philippines. C'est à l'une d'elles que nous avons confié, il y a quelques mois, la direction de cette province.

C'est encore au temps de sa fondatrice que l'institut fut introduit au Japon. Établi d'abord dans la région de Kagoshima en 1926, il passa en 1930 dans celle de Sendai. Là aussi il subit les dérangements de la guerre : ses missionnaires furent toutes rapatriées. Dès la fin des hostilités, l'évêque de Sendai les invita à reprendre leurs positions d'avantguerre. Présentement, elles sont réparties entre les écoles de Koriyama, de Wakamatsu et de Tokyo. C'est dans la capitale qu'ont été érigés le noviciat et un foyer pour étudiantes avec des activités connexes. A Koriyama, les søurs se dévouent auprès des orphelines.

Alors que la dernière guerre paralysait les missions de la communauté en Orient, une invitation de $\mathrm{M}^{\mathrm{gr}}$ Louis Collignon, o.m.i., évêque des Cayes, nous fit pénétrer aux Antilles. Haïti accueillit de nombreux groupes de nos missionnaires; quinze établissements sont disséminés du nord au sud de l'île et servent de cadres aux activités apostoliques les plus variées.

A Cuba, quand fut instauré le régime castriste, la communauté a dû céder au nouveau parti les sept * colegios > où elle dispensait l'enseignement. N'ayant plus de résidences, les sours revinrent au pays et, peu après, la plupart des rapatriées se réorientèrent vers l'Amérique du Sud. Cependant, à la demande du nonce apostolique, quelques sœurs demeurèrent à Cuba pour l'enseignement catéchétique; une partie de l'ancien séminaire des prêtres des Missions Étrangères à Colon les abrite en- 
core. Un noviciat a même été ouvert et a commencé à fournir des recrues cubaines.

A Hong Kong, on a repris en 1947 les activités d'avant-guerre. Trois écoles de quelques milliers d'élèves suppléent pour une part à l'insuffisance des établissements scolaires catholiques dans cette ville surpeuplée.

C'est en 1948 que notre communauté a été introduite en Afrique. Dans le Malawi et la Zambie, elle a onze secteurs d'évangélisation et d'œuvres sociales où l'on est sans cesse à l'écoute des besoins locaux. Nous sommes présentes en Afrique francophone par 45 des nôtres dispersées dans cinq écoles en république malgache.

Nos missions dernières-nées sont en Amérique latine : en excluant Haïti déjà mentionné, nous en trouvons trois au Pérou, quatre en Bolivie, deux au Chili; l'Amérique centrale compte deux maisons au Guatemala.

Nous faisons grâce à nos auditeurs de l'énumération fastidieuse des activités exercées par nos missionnaires dans chacune des régions mentionnées... Ces activités sont conditionnées par les directives de l'autorité diocésaine, les besoins des peuples et des Églises locales.

Après 69 ans d'existence, notre congrégation compte 984 sœurs professes, 23 novices et 15 postulantes réparties en neuf provinces dans 94. maisons situées en quinze pays différents.

\section{CONCLUSION}

Notre mot final sera à l'adresse de la Société cinquantenaire :

Qu'elle vive pendant des siècles... organisme dynamique au service de l'Église missionnaire ! Que ses fils marchent toujours dans le sillage de foi et de courage intrépides des devanciers de la première heure, réalisant ainsi l'attente des évêques fondateurs de cette Société des Missions Étrangères du Québec.

Mère Madeleine LoRANGER, m.i.c., supérieure générale. 